\title{
Donating Otx2 to support neighboring neuron survival
}

\author{
Hyoung-Tai Kim ${ }^{1}$, Alain Prochiantz ${ }^{2}$ E Jin Woo Kim ${ }^{1, *}$ \\ ${ }^{1}$ Department of Biological Sciences, Korea Advanced Institute of Science and Technology (KAIST), Daejeon 34141, Korea, ${ }^{2}$ Center for \\ Interdisciplinary Research in Biology (CIRB), College de France, Paris, France
}

\begin{abstract}
Mutations of orthodentricle homeobox 2 (OTX2) in human and mice often cause retinal dystrophy and nyctalopia, suggesting a role of OTX2 in mature retina, in addition to its functions in the development of the eye and retina. In support of this, the number of bipolar cells in Otx2 ${ }^{+/-}$post-natal mouse retina was found to be significantly lower than normal. Degeneration of the cells becomes greater as the mice age, leading to the loss of vision. Especially, the type-2 OFF-cone bipolar cells, which do not express Otx2 mRNA but carry Otx2 protein, are most sensitive to Otx2 haplodeficiency. Interestingly, this bipolar cell subpopulation imports Otx2 protein from photoreceptors to protect itself from glutamate excitotoxicity in the dark. Moreover, in the bipolar cells, the exogenous Otx2 relocates to the mitochondria to support mitochondrial ATP synthesis. This novel mitochondrial activity of exogenous Otx2 highlights the therapeutic potential of Otx2 protein transduction in retinal dystrophy. [BMB Reports 2016; 49(2): 69-70]
\end{abstract}

OTX2 is a homeodomain transcription factor that supports the development of fore- and midbrain compartments (Simeone et al (2002) Curr Opin Genet Dev 12: 409-415). Headless Otx $2^{-/-}$mice fail to develop beyond the early embryonic stage, a function that is conserved from arthropods to mammals including humans. However, the heterozygous loss of OTX2 is not life threatening, but causes multiple developmental defects in human. The mouse tissue most sensitive to Otx2 haplodeficiency is the eye, in which Otx2 is expressed in the embryonic optic neuroepithelium, and then later in the retinal pigment epithelium (RPE), bipolar cells and photoreceptors.

*Corresponding author. E-mail: jinwookim@kaist.ac.kr

http://dx.doi.org/10.5483/BMBRep.2016.49.2.006

Received 11 January 2016

Keywords: Glutamate excitotoxicity, Intercellular protein transfer, Mitochondria, Otx2, Retina

Abbreviations: Otx2, orthodentricle homeobox 2; RGC, retinal ganglion cell

Perspective to: Hyoung-Tai Kim et al., Mitochondrial Protection by Exogenous Otx2 in Mouse Retinal Neurons. Cell Reports (2015) 13(5), 990-1002. DOI, 10.1016/j.celrep.2015.09.075
In contrast to heterozygous OTX2 mutations that led to a broad range of morphological defects in the human eye, a majority of Otx2 heterozygote mice exhibited retinal dystrophy with normal eye size (Bernard et al (2014) Hum Mol Genet 23: 1742-1753, DOI. $10.1093 / \mathrm{hmg} / \mathrm{ddt} 562$ ), suggesting that unidentified OTX2 heterozygous mutations might be associated with human retinal dystrophy without any accompanying gross eye defect. Bipolar cells, which relay the response of photoreceptors to retinal ganglion cells (RGCs), showed a significant loss in the dystrophic Otx2 $2^{+/-}$mouse retina. Especially, the type-2 OFF-cone bipolar cells completely disappeared in the Otx $2^{+/-}$adult mouse retina, although they developed normally in the mice. Strikingly, the type-2 OFF-cone bipolar cells possessed Otx2 protein, in the absence of any Otx2 mRNA expression. This implicates that Otx2 in the bipolar cells originates from external Otx2-producing sources.

Using two conditional knock-out mouse models, namely $\mathrm{Otx} 2^{\mathrm{ff} f} ; \mathrm{Crx}-\mathrm{CreER}^{\mathrm{t} 2}$ and Otx2 $2^{\mathrm{fff}}$;Chx10-CreER ${ }^{\mathrm{t} 2}$, which express $\mathrm{CreER}^{\mathrm{t} 2}$ recombinases in photoreceptors and bipolar cells respectively, we proved that the photoreceptors provide Otx2 protein to type-2 OFF cone bipolar cells. Upon deletion of Otx2 in the photoreceptors, levels of Otx2 were decreased not only in type-2 OFF-cone bipolar cells but also in rod bipolar cells, suggesting that Otx2 uptake takes place in all bipolar cells. However, deleting Otx2 in most bipolar cells in the $\mathrm{Otx} 2^{\mathrm{fff}}$; Chx10-CreER ${ }^{t 2}$ mouse retina did not modify the Otx2 content of type-2 OFF-cone bipolar cells, indicating that lateral transfer between the bipolar cells is negligible.

Intercellular transfer of Otx2 has previously been identified in the visual cortex, where Otx2 specifically accumulates in parvalbumin (PV)-positive GABAergic interneurons, starting at the onset of the critical period for binocular visual maturation (Sugiyama et al (2008) Cell 134, 508-520). Although the choroid plexus was identified as an Otx2 source in cortical PV neurons (Spatazza et al (2013) Cell Rep 3: 1815-1823, DOI, 10.1016/j.celrep.2013.05.014), other sources may also co-exist. In addition, several reports demonstrated that Otx2 injected in the vitreal space can gain access to several cell types in the retina, as well as in the cortex (Sugiyama et al (2008) Cell 134: 508-520, DOI, 10.1016/j.cell.2008; Torero lbad et al (2011) J Neurosci 31: 5495-5503. doi: 10.1523/JNEUROSCI.0187-11. 2011). Accordingly, injecting Otx2 into the vitreal space of 2 week-old mouse eyes blocked the bipolar cell degeneration and retinal dystrophy in adult Otx $2^{+/-}$mice. 

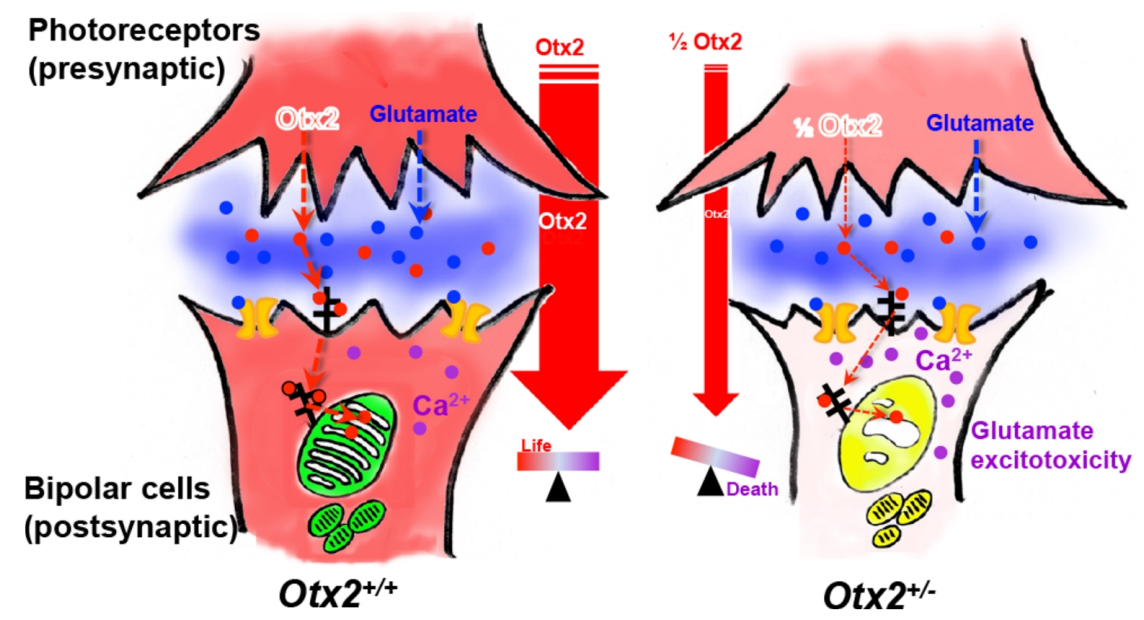

Fig. 1. Neuroprotection by exogenous Otx2 in retinal bipolar cells. Glutamate is released from photoreceptors in the dark, to activate glutamate receptors in the postsynaptic OFF-cone bipolar cells. Consequent elevation of intracellular calcium in the OFF-cone bipolar cells often induces excitotoxicity to the cells. Otx2 expressed in the photoreceptors is released into the synaptic cleft and captured by the OFF-cone bipolar cells for subsequent penetration into the cells. Otx2 in the OFF-cone bipolar cells moves into the mitochondria to facilitate mitochondrial activity, which is necessary to prevent the neurons from glutamate excitotoxicity. In Otx $2^{+/-}$mice, the amount of Otx2 transferred is decreased below a critical level, resulting in the mitochondrial dysfunction and degeneration of the neurons.

Despite its neuroprotective activity, the role of exogenous Otx2 in bipolar cells remains to be explored. We have tested the hypothesis of its activity at the level of mitochondria. In contrast to its strong nuclear enrichment in photoreceptors and bipolar cells, exogenous Otx2 in mouse RGC was located in the cytoplasm. Moreover, Otx2 was added to cultured retinal cells or injected into the vitreal space associated with mitochondria, suggesting that external Otx2 can be delivered to this organelle following the internalization. In the mitochondria, Otx2 interacted with mitochondrial ATP synthase subunit a1 (Atp5a1), a F1 catalytic core component of ATP synthase comprising the mitochondrial oxidative phosphorylation (OXPHOS) complex V. Otx2 not only interacted with Atp5a1, but could also integrate into the mitochondrial ATP synthase complex and supported the ATP synthesis to keep up with demands of ATP-dependent post-synaptic events.

One of the ATP-dependent post-synaptic events addressed in this study was protection against glutamate excitotoxicity. Vertebrate photoreceptors have a unique property to spontaneously release glutamate in the absence of light stimulus. Therefore, OFF-type bipolar cells are persistently depolarized by photoreceptor glutamate release in the dark. Since the OFF-type bipolar cells were more sensitive than the ON-type bipolar cells to Otx2 haplodeficiency, we hypothesized that the photoreceptors provide Otx2 to their post-synaptic OFFcone bipolar cells, and thus protect them from glutamate excitotoxicity. In support of this hypothesis, we found that the OFF-cone bipolar cells die in dark-reared Otx $2^{+/-}$mice, while the ON-type bipolar cells remain unaffected. All in all, these results suggest that reduced levels of Otx2 in Otx2 $2^{+1-}$ mouse photoreceptors, and the ensuing decrease in Otx2 transfer to OFF-cone bipolar cells, impaired the bipolar cell resistance to excitotoxicity (Fig. 1).

This study provides a molecular interpretation for the retinal dystrophy observed in OTX2 haplodeficiency in human patients. It also suggests that OTX2 could be used as a therapeutic protein in eye diseases. Similar to the Engrailed homeoprotein that has been used as a therapeutic protein in mouse models of Parkinson Disease (Alvarez-Fischer et al (2011) Nat Neurosci 14: 1260-1266, DOI, 10.1038/nn.2916), it is tempting to propose that similar neuroprotective functions could be carried by other members of the homeodomain transcription factor, including OTX2.

\section{ACKNOWLEDGEMENTS}

This work was supported by grants from National Research Foundation of Korea (NRF-2009-00424). 